\title{
Sigma-models with complex homogeneous target spaces
}

\author{
Dmitri Bykov ${ }^{1,2, \star}$ \\ ${ }^{1}$ Max-Planck-Institut für Gravitationsphysik, Albert-Einstein-Institut, \\ Am Mühlenberg 1, D-14476 Potsdam-Golm, Germany \\ ${ }^{2}$ Steklov Mathematical Institute of Russ. Acad. Sci., \\ Gubkina str. 8, 119991 Moscow, Russia
}

\begin{abstract}
We review the recently proposed class of $\sigma$-models with complex homogeneous target spaces, whose equations of motion admit zero-curvature representations.
\end{abstract}

\section{The models}

The action of a $\sigma$-model describing maps $X$ from a 2D worldsheet $\mathscr{C}$ to a target space $\mathscr{M}$ with metric $h$ is given by

$$
\mathcal{S}=\frac{1}{2} \int_{\mathscr{C}} d^{2} z h_{i j}(X) \partial_{\mu} X^{i} \partial_{\mu} X^{j}
$$

Its critical points $X(z, \bar{z})$ are called harmonic maps. We will be interested in the case when the target space $\mathscr{M}$ is homogeneous: $\mathscr{M}=G / H, G$ compact and semi-simple. We will use the following standard decomposition of the Lie algebra $g$ of $G$ :

$$
\mathfrak{g}=\mathfrak{h} \oplus \mathfrak{m},
$$

where $\mathfrak{m} \perp \mathfrak{h}$ with respect to the Killing metric on $\mathfrak{g}$. For a reductive homogeneous space one has the following relations:

$$
\begin{array}{lll}
{[\mathfrak{h}, \mathfrak{h}] \subset \mathfrak{h}} & \Rightarrow & \mathfrak{h} \text { is a subalgebra of } \mathfrak{g} \\
{[\mathfrak{h}, \mathfrak{m}] \subset \mathfrak{m}} & \Rightarrow \quad \mathfrak{m} \text { is a representation of } \mathfrak{h}
\end{array}
$$

A homogeneous space $G / H$ is called symmetric if

$$
[\mathfrak{m}, \mathfrak{m}] \subset \mathfrak{h}
$$

Equivalently, there exists a $\mathbb{Z}_{2}$-grading on $\mathfrak{g}$, i.e. a Lie algebra homomorphism $\sigma$ of $\mathfrak{g}$, such that $\sigma(a)=a$ for $a \in \mathfrak{h}$ and $\sigma(b)=-b$ for $b \in \mathfrak{m}$.

The action of a $\sigma$-model with homogeneous target space $G / H$ is globally invariant under the Lie group $G$. Therefore, there exists a conserved Noether current $K^{\mu} \in \mathfrak{g}$ :

$$
\partial_{\mu} K^{\mu}=0
$$

^e-mail:dmitri.bykov@aei.mpg.de,dbykov@mi.ras.ru 
Since the group $G$ acts transitively on its quotient space $G / H$, the equations of motion are in fact equivalent to the conservation of the current.

It was observed by Pohlmeyer [1] that in the case when the target space is symmetric, the current $K$ is, moreover, flat (when viewed as a one-form and with proper normalization):

$$
d K-K \wedge K=0
$$

To get an idea, why this can be the case, recall that the Maurer-Cartan equation has the solution

$$
K=-g^{-1} d g, \quad g \in G
$$

What is the relation between $g$ and a point in the configuration space $[\tilde{g}] \in G / H$ ? The answer is given by Cartan's embedding $G / H \hookrightarrow G$ :

$$
g=\widehat{\sigma}(\tilde{g}) \tilde{g}^{-1}
$$

$\widehat{\sigma}$ is a Lie group homomorphism induced by the Lie algebra involution $\sigma$.

Another observation of Pohlmeyer was that the two conditions

$$
\begin{array}{cr}
d * K=0 \quad \text { (Conservation) } \\
d K-K \wedge K=0 \quad \text { (Flatness) }
\end{array}
$$

may be rewritten as an equation of flatness of a connection

$$
A_{u}=\frac{1+u}{2} K_{z} d z+\frac{1+u^{-1}}{2} K_{\bar{z}} d \bar{z}
$$

where we have decomposed the current $K=K_{z} d z+K_{\bar{z}} d \bar{z}$. We have

$$
d A_{u}-A_{u} \wedge A_{u}=0
$$

This leads to an associated linear system (Lax pair)

$$
\left(d-A_{u}\right) \Psi=0
$$

The existence of a linear system described above is often a sufficient condition for the classical integrability of the model. The linear system was used in [2] to solve the equations of motion for the principal chiral model (target space $G$ ), with worldsheet $\mathbf{C} \mathbf{P}^{1}$. A more rigorous approach was developed in [3]. Solutions of the e.o.m. for $\sigma$-models with symmetric target spaces may be obtained by restricting the solutions of the principal chiral model. These constructions could not be directly generalized to the case of homogeneous, but not symmetric target spaces (in particular, because there is no Cartan involution).

We will consider a different class of models, with target spaces $\mathcal{M}$ of the following type:

- $\mathcal{M}=G / H$ is a homogeneous space; for simplicity we take $G$ compact and semi-simple

$$
\mathfrak{g}=\mathfrak{h} \oplus \mathfrak{m}, \quad[\mathfrak{h}, \mathfrak{h}] \subset \mathfrak{h}, \quad[\mathfrak{h}, \mathfrak{m}] \subset \mathfrak{m}
$$

- $\mathcal{M}$ has an integrable $G$-invariant complex structure $\mathscr{I}$

$$
\mathfrak{m}=\mathfrak{m}_{+}+\mathfrak{m}_{-}, \quad\left[\mathfrak{h}, \mathfrak{m}_{ \pm}\right] \subset \mathfrak{m}_{ \pm}, \quad\left[\mathfrak{m}_{ \pm}, \mathfrak{m}_{ \pm}\right] \subset \mathfrak{m}_{ \pm}
$$

Here $\mathfrak{m}_{ \pm}$may be thought of as the holomorphic/anti-holomorphic tangent spaces to $\mathcal{M}$, i.e. $\mathscr{I} \circ \mathrm{m}_{ \pm}=$ $\pm i \mathrm{~m}_{ \pm}$. 
- The Killing metric $h$ is Hermitian (i.e. of type $(1,1))$ w.r.t. $\mathscr{I}$

$$
h\left(\mathfrak{m}_{ \pm}, \mathrm{m}_{ \pm}\right)=0
$$

Complex homogeneous spaces were classified in [4] a long time ago. They are toric bundles over flag manifolds. Consider for simplicity the case of $G=S U(N)$. Then the relevant manifolds are of the form

$$
\mathcal{M}=\frac{S U(N)}{S\left(U\left(n_{1}\right) \times \ldots \times U\left(n_{m}\right)\right)}, \quad \sum_{i=1}^{m} n_{i} \leq N,
$$

One has to choose the integers $n_{i}$ in such a way that $\mathcal{M}$ is even-dimensional. If $\sum_{i=1}^{m} n_{i}=N$, this is the manifold of partial flags in $\mathbf{C}^{N}$. Otherwise it is a $U(1)^{2 s}$-bundle over a flag manifold, where $2 s=N-\sum_{i=1}^{m} n_{i}$.

Given a homogeneous space of the type just described, one can introduce the action of the model [5]:

$$
\begin{aligned}
& \mathcal{S}=\int_{\mathscr{C}} d^{2} z\|\partial X\|^{2}+\int_{\mathscr{C}} X^{*} \omega= \\
& =\int_{\mathscr{C}} d^{2} z\left(h_{i j} \partial_{\mu} X^{i} \partial_{\mu} X^{j}+\epsilon_{\mu \nu} \omega_{i j} \partial_{\mu} X^{i} \partial_{\nu} X^{j}\right),
\end{aligned}
$$

where $\omega=h \circ \mathscr{I}$ is the Kähler form. Note, however, that, in general, the metric $h$ is not Kähler, hence the form $\omega$ is not closed: $d \omega \neq 0$. Therefore the second term in the action contributes to the e.o.m.!

Let $K$ be the Noether current constructed using the above action. As we already discussed, the e.o.m. are equivalent to its conservation:

$$
d * K=0
$$

The key observation is that, for the models considered, it is also flat:

$$
d K-K \wedge K=0
$$

These two equations mean, in essence, that the described models are sub-models of the principal chiral model (PCM). In particular, the solutions of these models are a subset of solutions of the PCM. The Lax pair representation can be constructed in parallel with the Pohlmeyer procedure.

Symmetric spaces of the group $S U(N)$ are the Grassmannians

$$
\mathbb{G}_{n \mid N}:=\frac{S U(N)}{S(U(n) \times U(N-n))}
$$

In this case the canonical one-parametric family of flat connections has the form

$$
\widetilde{A}_{\lambda}=\frac{1-\lambda}{2} \widetilde{K}_{z} d z+\frac{1-\lambda^{-1}}{2} \widetilde{K}_{\bar{z}} d \bar{z}
$$

where $\widetilde{K}$ is the canonical Noether current, i.e. the one constructed using the standard action

$$
\mathcal{S}=\frac{1}{2} \int_{\mathscr{C}} d^{2} z h_{i j}(X) \partial_{\mu} X^{i} \partial_{\mu} X^{j}
$$


The models, which we described above, feature an additional term in their action: $\int_{\mathscr{C}} X^{*} \omega$, the integral of the Kähler form. Therefore the Noether current $K$ defined using this action will be different from $\widetilde{K}$, the difference being a 'topological' current:

$$
K=\widetilde{K}+* d M
$$

Nevertheless both $K$ and $\widetilde{K}$ are flat. The one-parametric family of connections constructed using the current $K$ has the form

$$
A_{u}=\frac{1+u}{2} K_{z} d z+\frac{1+u^{-1}}{2} K_{\bar{z}} d \bar{z}
$$

A natural question arises: How are $\widetilde{A}_{\lambda}$ and $A_{u}$ related? The answer is: $\widetilde{A}_{\lambda}$ and $A_{u}$ are related by a gauge transformation $\Omega$ :

$$
\widetilde{A_{\lambda}}=\Omega A_{u} \Omega^{-1}-\Omega d \Omega^{-1}
$$

$\Omega$ can be written out explicitly ( $g$ is the 'dynamical' group element):

$$
\Omega=g \Lambda g^{-1}, \quad \text { where } \Lambda=\operatorname{diag}(\underbrace{\lambda^{-1 / 2}, \ldots, \lambda^{-1 / 2}}_{n}, \underbrace{\lambda^{1 / 2}, \ldots, \lambda^{1 / 2}}_{N-n})
$$

Rather important is the nontrivial relation between the spectral parameters:

$$
\lambda=u^{1 / 2}
$$

This relation may be confirmed by analyzing the limiting behavior of the holonomies of the connection as $u \rightarrow 0$ (such analysis can be borrowed from [6]).

\section{An example. The flag manifold.}

As an example let us consider one of the simplest homogeneous, but non-symmetric spaces - the flag manifold

$$
\mathcal{F}_{3}=\frac{U(3)}{U(1)^{3}}
$$

It is the space of ordered triples of lines through the origin in $\mathbb{C}^{3}$, and can be parametrized by three orthonormal vectors $u_{i}, i=1,2,3, \bar{u}_{i} \circ u_{j}=\delta_{i j}$, modulo phase rotations: $u_{k} \sim e^{i \alpha_{k}} u_{k}$.

To formulate the model, we need to pick a particular complex structure on $\mathcal{F}_{3}$. The (co)tangent space to $\mathcal{F}_{3}$ is spanned at each point by the one-forms

$$
J_{i j}:=u_{i} \circ d \bar{u}_{j}, \quad i \neq j
$$

One can pick any three non-mutually conjugate one-forms and define the action of the complex structure operator $\mathscr{I}$ on them:

$$
\mathscr{I} \circ J_{12}= \pm i J_{12}, \quad \mathscr{I} \circ J_{23}= \pm i J_{23}, \quad \mathscr{I} \circ J_{31}= \pm i J_{31}
$$

Altogether there are $2^{3}=8$ possible choices, so that there are 8 invariant almost complex structures. However, only 6 of them are integrable.

Pick the integrable complex structure $\mathscr{I}$, in which $J_{12}, J_{13}, J_{23}$ are holomorphic one-forms. Then the action can be written as [7]

$$
\mathcal{S}=\int d^{2} z\left(\left|\left(J_{12}\right)_{\bar{z}}\right|^{2}+\left|\left(J_{13}\right)_{\bar{z}}\right|^{2}+\left|\left(J_{23}\right)_{\bar{z}}\right|^{2}\right)
$$


The e.o.m. are:

$$
\mathscr{D}_{z}\left(J_{12}\right)_{\bar{z}}=0, \quad \mathscr{D}_{z}\left(J_{31}\right)_{\bar{z}}=0, \quad \mathscr{D}_{z}\left(J_{23}\right)_{\bar{z}}=0 \quad \text { and c.c. ones }
$$

From the action (16) it is clear that the holomorphic curves defined by $\left(J_{12}\right)_{\bar{z}}=\left(J_{13}\right)_{\bar{z}}=\left(J_{23}\right)_{\bar{z}}=0$ minimize the action, hence are solutions of the e.o.m. From (17) it follows that $\left(J_{12}\right)_{\bar{z}}=\left(J_{31}\right)_{\bar{z}}=$ $\left(J_{23}\right)_{\bar{z}}=0$ is a solution as well. This defines a curve, holomorphic in a different, non-integrable almost complex structure $I$. We have seen that the curves, holomorphic in at least two different almost

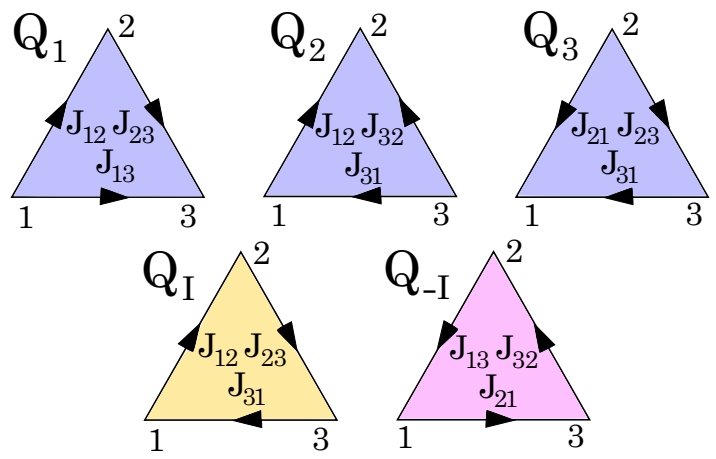

Figure 1. The triangles indicate the complex structures, whose associated holomorphic curves are solutions of the $\sigma$-model. The three top triangles correspond to integrable complex structures, whereas the two lower ones correspond to the non-integrable ones.

complex structures, satisfy the e.o.m. As we discussed, there are 8 almost complex structures on the flag manifold. Are there any other holomorphic curves that still solve the e.o.m.? The answer is positive. The relevant complex structures are shown in Fig. 1.

We have already discussed why the $Q_{I}$-holomorphic curves and $Q_{1}$-holomorphic curves satisfy the e.o.m. To see why the $Q_{2}$ - and $Q_{3}$-holomorphic curves satisfy the e.o.m., one should note that the differences between the respective Kähler forms are closed forms, i.e. for example $\omega_{1}-\omega_{2}=\Omega_{t o p}$ with $d \Omega_{\text {top }}=0$. Therefore the two actions $\mathcal{S}_{1}$ and $\mathcal{S}_{2}$ differ by a topological term:

$$
\mathcal{S}_{1}-\mathcal{S}_{2}=\int_{\mathscr{C}} \Omega_{t o p}
$$

This leads to an interesting bound on the instanton numbers of the holomorphic curves. To see this, note that the flag manifold may be embedded as

$$
i: \mathcal{F}_{3} \hookrightarrow \mathbf{C P}^{2} \times \mathbf{C} \mathbf{P}^{2} \times \mathbf{C P}^{2}
$$

The second cohomology $H^{2}\left(\mathcal{F}_{3}, \mathbb{R}\right)=\mathbb{R}^{2}$ can be described via the pull-backs of the Fubini-Study forms of the $\mathbf{C} \mathbf{P}^{2}$ 's, and the corresponding instanton numbers are $n_{i}=\int_{\mathscr{C}} i^{*}\left(\Omega_{F S}^{(i)}\right), i=1,2,3$.

These are subject to the condition

$$
n_{1}+n_{2}+n_{3}=0
$$

The bounds on the topological numbers $n_{i}$ for the holomorphic curves, which follow from the nonnegativity of the actions $\mathcal{S}_{i}$, are shown in Fig. 2. 


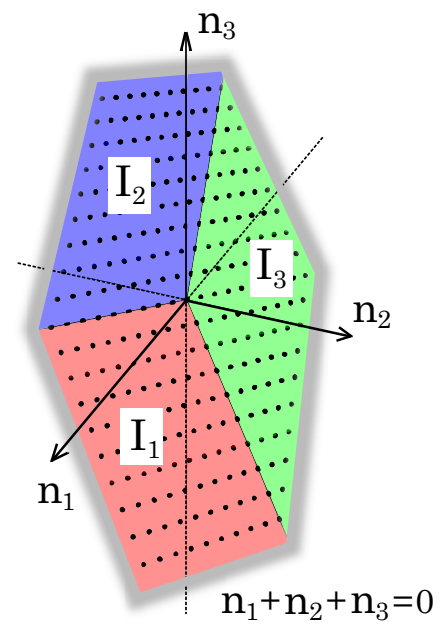

Figure 2. Admissible instanton numbers for various complex structures on $\frac{U(3)}{U(1)^{3}}$ are shown by black dots.

In [8] we were able to construct all solutions to the e.o.m. of the flag manifold $\sigma$-model for the case when the worldsheet is a sphere, $\mathscr{C}=\mathbf{C P}$. In that case all solutions to the e.o.m. are parametrized by the following data:

- One of the projections $\pi_{i}: \mathcal{F}_{3} \rightarrow\left(\mathbf{C P}^{2}\right)_{i}, \quad i=1,2,3$

- A harmonic map $v_{\text {har }}: \mathbf{C} \mathbf{P}^{1} \rightarrow\left(\mathbf{C P}^{2}\right)_{i}$ to the base of the projection

- A holomorphic map $w_{\text {hol }}: \mathbf{C} \mathbf{P}^{1} \rightarrow \mathbf{C} \mathbf{P}^{1}$ to the fiber of the projection, .

For every triple $\left(i, v_{h a r}, w_{h o l}\right)$ there exists a solution of the e.o.m., and all solutions are obtained in this way [8]. The crucial point is that the harmonic maps to the base manifold $\mathbf{C P}^{2}$ are known explicitly [9] (and the holomorphic maps $\mathbf{C} \mathbf{P}^{1} \rightarrow \mathbf{C} \mathbf{P}^{1}$ are just rational functions).

\section{Discussion}

It has been known for a long time that the e.o.m. of $\sigma$-models with symmetric target spaces admit zero-curvature representations - this fact is the cornerstone of integrability of these models. In our recent works we have considered modified $\sigma$-models with complex homogeneous target spaces, not necessarily symmetric ones, for which there exist Lax pairs as well [5]. This class of models was reviewed above in section 1. We have also considered in detail a concrete example of such model, namely the case when the target space is the flag manifold $\frac{U(3)}{U(1)^{3}}$ [7]. When the worldsheet is a sphere $\mathbf{C} \mathbf{P}^{1}$, all solutions of the e.o.m. have been constructed [8]. These results were summarized in section 2. The crucial test of integrability of the proposed models would lie in the construction of classical solutions for the case when the worldsheet is a torus $S^{1} \times S^{1}$ (as was done in [6] for $\mathcal{M}=S U(2)$ ). Other directions for future research include supersymmetrization of the models and the clarification of the role of Lie algebra gradings (such as $\mathbb{Z}_{2}$ for symmetric spaces, or $\mathbb{Z}_{4}$ for semisymmetric spaces). 
Acknowledgements. I would like to thank the organizers of the conference 'Quarks 2016' for the inspiring and productive atmosphere. I am indebted to Prof. A.A.Slavnov and to my parents for support and encouragement. My work was supported in part by the grant RFBR 14-01-00695-a.

\section{References}

[1] K. Pohlmeyer, Communications in Mathematical Physics 46, 207 (1976)

[2] V.E. Zakharov, A.V. Mikhailov, Sov. Phys. JETP 47, 1017 (1978), [Zh. Eksp. Teor. Fiz.74,1953(1978)]

[3] K. Uhlenbeck, Journal of Differential Geometry 30, 1 (1989)

[4] H.C. Wang, Amer. J. Math. 76, 1 (1954)

[5] D. Bykov, Phys. Lett. B 760C, 341 (2016), 1605.01093

[6] N.J. Hitchin, Journal of Differential Geometry 31, 627 (1990)

[7] D. Bykov, Nucl. Phys. B894, 254 (2015), 1412 . 3746

[8] D. Bykov, Nucl. Phys. B902, 292 (2016), 1506.08156

[9] A.M. Din, W.J. Zakrzewski, Nucl. Phys. B174, 397 (1980) 